\title{
Incoherent Effects of Electron Clouds in Proton Storage Rings
}

\author{
E. Benedetto, ${ }^{1,2}$ G. Franchetti, ${ }^{3}$ and F. Zimmermann ${ }^{1}$ \\ ${ }^{1}$ CERN, Geneva, Switzerland \\ ${ }^{2}$ Politecnico Torino, Italy \\ ${ }^{3}$ GSI, Darmstadt, Germany \\ (Received 16 March 2006; published 21 July 2006)
}

\begin{abstract}
Electron clouds in the beam pipe of high-energy proton or positron storage rings can give rise to significant incoherent emittance growth, at densities far below the coherent-instability threshold. We identify two responsible mechanisms: namely, (1) a beam particle periodically crosses a resonance and (2) a beam particle periodically crosses a region of the bunch where its motion is linearly unstable. Formation of halo or beam-core blow up, respectively, are the result. Key ingredients for both processes are synchrotron motion and electron-induced tune shift. The mechanisms considered provide a possible explanation for reduced beam lifetime and emittance growth observed at several operating accelerators. Similar phenomena are likely to occur in other two-stream systems.
\end{abstract}

DOI: 10.1103/PhysRevLett.97.034801

In the vacuum chamber of charged-particle storage rings, electrons are produced by a number of processes, such as gas ionization, photoemission, and secondary emission [1]. Above a certain electron-density threshold, the electrons induce fast multibunch [2] or single-bunch instabilities $[3,4]$ of positively charged beams. However, electron-cloud effects below the threshold of coherent instability are also a concern, in particular, for proton storage rings like the Large Hadron Collider (LHC), now under construction, where synchrotron-radiation damping is effective only after many hours, and small perturbations can lead to significant long-term emittance growth.

Experience at operating storage rings indeed hints at possible incoherent effects at "moderate" electron density: The lifetime of the LHC proton beam in the CERN Super Proton Synchrotron (SPS) is limited to 5-20 min at the injection momentum of $26 \mathrm{GeV} / c$ [5]. The lifetime decreases along the bunch train in a pattern which closely resembles the measured buildup pattern of the electron cloud [6]. At the KEK $B$ factory a clear threshold in the beam current is observed, above which the vertical beam size steeply rises with further increasing current [7]. Remarkably, already below the threshold the measured beam size increases with beam current, though more gradually [7]. Also at RHIC an electron cloud causes emittance growth and beam loss [8]. Similar effects were seen at the Tevatron for 19-ns bunch spacing, without any sign of coherent beam motion [9], still requiring further verification.

A detailed computer model of the beam-electron interaction is provided by particle-in-cell (PIC) computer simulations of a proton bunch passing through an electron cloud, as implemented, e.g., in the code HEADTAIL [10]. The beam-electron interaction is usually concentrated in a finite number (1-100) of "interaction points" around the model storage ring, with the effective electron density properly chosen to yield, e.g., the same tune shift as expected from a realistic distribution of electrons around
PACS numbers: 29.27.Bd, 05.45.Pq, 52.35.Qz, 52.40.Mj

the ring. A small number of interaction points does not properly resolve the actual betatron motion, however, and, in particular, it can lead to the artificial excitation of resonances. For the standard LHC simulation parameters (see Tables I and II in [11]), the emittance growth below the coherent-instability threshold is found to be roughly independent of the number of macroparticles representing the electrons and the proton bunch, but to strongly depend on the number of electron-beam interaction points. Also, this emittance growth is nearly the same if one either treats the motion of both protons and electrons dynamically, or if one follows the motion of individual protons moving through a static or "frozen" electron cloud. In the latter case, the electric potential of the cloud is computed only during the first bunch passage, and the same potential is then assumed for all later beam-cloud interactions. The frozen cloud is a good approximation if coherent instabilities are absent.

Figure 1 illustrates the simulated evolution of the electron-cloud density $\rho_{e}$ during a bunch passage, for an initially uniform electron distribution, in a field-free region of the LHC. Shown is the average local density inside a circle of variable radius as a function of position $z$ along the bunch. The electrons primarily move in the transverse plane. Electrons initially located in the nearly linear portion of the beam force, i.e., within the rms beam volume, perform approximately linear oscillations in the beam potential. The motion of these electrons yields a high local electron density after their first quarter oscillation, which then repeats every half electron oscillation period. Superimposed on these linearly oscillating electrons is a gradual increase in the central electron density due to electrons which start farther away from the beam and which undergo nonlinear oscillations under the influence of the nonlinear beam field. At the center of the beam the electron density increases by more than 2 orders of magnitude compared with the initial uniform density. Towards the tail of the bunch, the potential of the beam decreases and some of the oscillating electrons may be released towards larger am- 


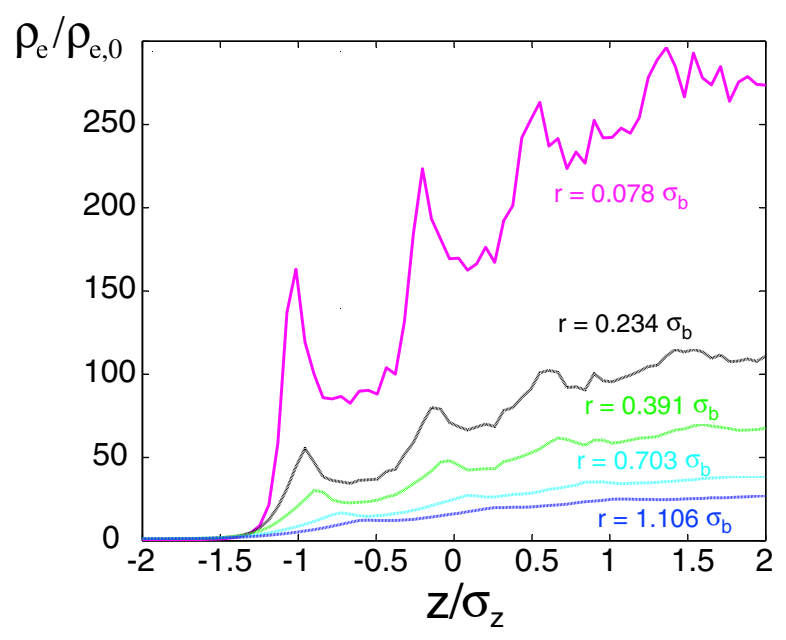

FIG. 1 (color online). Simulated average electron density inside a circle of variable radius $r$, with $\sigma_{b}$ denoting the rms beam size. The bunch tail is in the region $z>0$. The coordinate system is moving longitudinally with the bunch.

plitudes. As a result of the local electron-density enhancement ("pinch") during the bunch passage, the beam particles experience a large incoherent betatron tune shift, which increases towards the bunch tail. However, at all times the electron density remains small compared with the local proton density inside the bunch (about $10^{17} \mathrm{~m}^{-3}$ ), so that electron space-charge self-forces-optionally included in the simulation - may be neglected. The incoherent tune shift due to an electron cloud was first estimated by Furman and Zholents for the PEP-II $B$ factory [12], who found that it was moderate and that the electron density was roughly uniform in the transverse dimension inside the bunch. For the examples considered here, however, the electrons perform several oscillations during a bunch passage, leading to an electron distribution which is narrowly spiked in the transverse dimension, with an rms width $\sigma_{e}$ much smaller than the rms beam size $\sigma_{b}$, and to an incoherent tune shift at least 10 times larger than the coherent one which, in the SPS, was measured to be of order 0.010.02 .

The simulated emittance growth depends on the number of interaction points around the ring, which we attribute to the pertinent change in the strength of resonance excitation. More importantly, without synchrotron motion the emittance growth either vanishes or saturates at a low level, indicating that the longitudinal oscillations are an important ingredient.

The tune shift due to the electron cloud depends on the longitudinal coordinate with respect to the bunch center, $z$, and so does the detuning with amplitude. In consequence, resonance islands change their size and location as a function of $z$. Beam particles executing synchrotron motion may cross important resonances twice or 4 times during a synchrotron period. The particles can then be trapped inside a resonance island and, as the island position changes along the bunch, in the course of their synchrotron motion be transported to larger (or smaller) amplitudes, where they may become untrapped [13]. They can also scatter off a chaotic region near the resonance. Another description of this same process is in terms of modulational diffusion [14]. Namely, viewed in a stroboscopic frame (once per synchrotron period), the modulation introduces sideband resonances. Since the maximum (over all beam particles) incoherent tune shift $\Delta Q$ of order 0.1 is much larger than the modulation tune $Q_{m}$, which is equal to the synchrotron tune, about 0.001 , many sidebands overlap, forming a wide chaotic layer, in which strong diffusion can occur.

Evidence of modulational diffusion in the simulation is presented in Fig. 2, which shows the square of the horizontal oscillation amplitude for a single proton as a function of turn number. Periodic step changes occurring twice per synchrotron period are characteristic of resonance trapping and detrapping, or modulational diffusion. A similar mechanism was shown to be at play-both in simulations and experiments - for halo generation and beam loss due to space charge [15]. Compared with space charge, the electron cloud features no front-back symmetry, the transverse distribution is highly nonuniform, and the sign of the tune shift is opposite.

Since the weak-strong simulation, based on a frozen cloud, gives a good description of the emittance evolution below the fast-instability threshold, we have developed an alternative, faster computer model, where we employ an analytical description of the electric field instead of inferring it from electron macroparticles on a grid. For simplicity, at each longitudinal position $z$ along the bunch, the electron cloud is approximated by a distribution whose profile is Gaussian in the plane perpendicular to $z$ and whose $z$-dependent peak value and rms size may be inferred from an independent PIC simulation of a singlebunch passage through the electron cloud. This scheme avoids any inherent noise of the PIC calculation and is faster, opening up the way to simulating the effect of the real electron distribution all around the ring, using a much larger number of interaction points than is possible in the

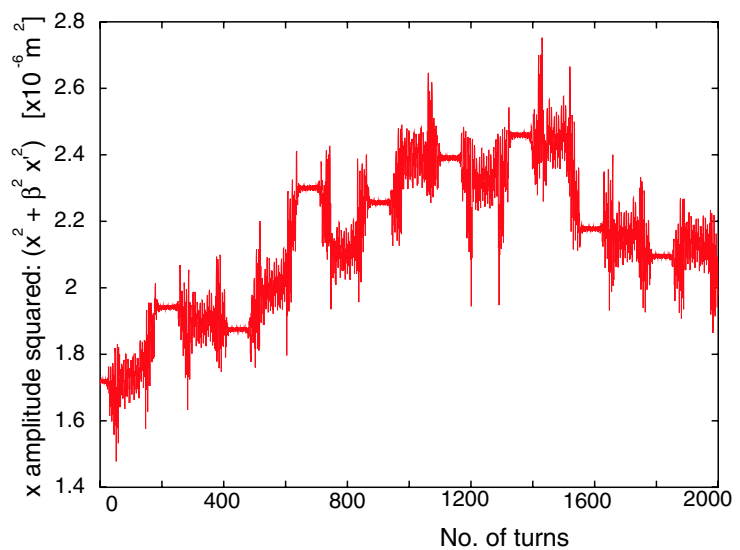

FIG. 2 (color online). Horizontal invariant of the unperturbed linear system for a proton at a large synchrotron amplitude as a function of turn number, from a simulation. 
PIC scheme. Although the realistic pinched electron distribution is not Gaussian, the analytical approximation allows us to explore the main features of the effect and it yields the correct field outside the core of the pinched electron cloud (a fraction of the beam size).

For the purpose of benchmarking the two types of simulations against each other, we chose the case of a single interaction point. First, we considered the artificial example of a static Gaussian electron distribution with $\sigma_{e}$ independent of $z$ and constant in time, and equal to the transverse rms beam size $\sigma_{b}$. The electron density was taken to increase linearly along the bunch, giving rise to a maximum incoherent tune shift of 0.1 , experienced by particles in the bunch tail. Figure 3(a) demonstrates that the results of the two simulations are nearly indistinguishable. We next modeled the field of the pinched electrons. We again used either the PIC code (which is accurate only up to the grid size of about a tenth of the beam size), or its approximation by a Gaussian with now varying central electron density $\rho_{e}(z)$ and rms transverse size $\sigma_{e}(z)$. In the latter approximation, we kept the product $\rho_{e} \sigma_{e}^{2}$ constant (which underestimates the total number of electrons inside the bunch as determined by the PIC code), and the peak electron density was set so as to obtain a maximum incoherent tune shift of 0.04 , which is experienced by beam particles at some longitudinal position $z$ along the bunch, as determined by the PIC simulation. The results for this case are displayed in Fig. 3(b). The two simulated curves are different, although the behavior remains qualitatively similar. The simulations in Fig. 3 are not meant to predict the actual emittance growth, but to validate the analytical model.

At high electron density, the simulations reveal halo formation of the proton beam in the case where $\sigma_{e}$ is
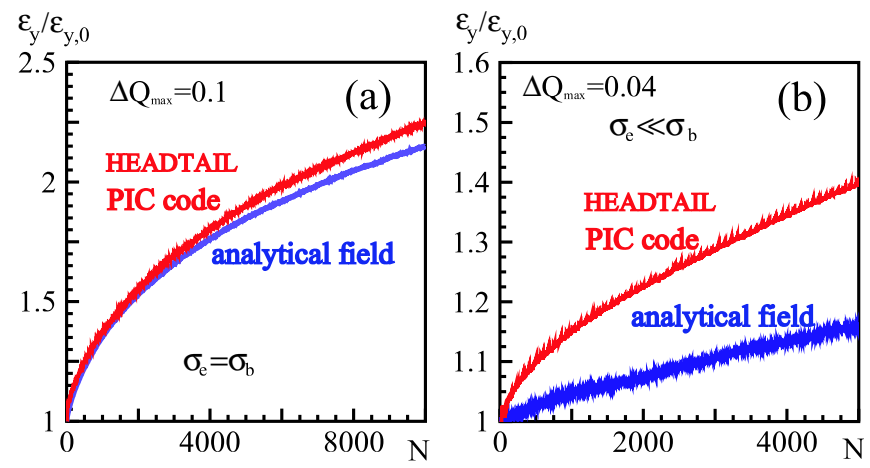

FIG. 3 (color online). Vertical emittance growth simulated by either the HEADTAIL PIC code or the means of an analytical field model (using $3 \times 10^{5}$ and $3 \times 10^{4}\left[10^{4}\right.$ in (b)] beam macroparticles, respectively) as a function of turn number, for a Gaussian electron cloud with constant size equal to the beam size and linearly increasing density, with a maximum tune shift of 0.1 (a), and for the real pinched distribution or its approximation with varying transverse size and oscillating electron density, for a maximum tune shift of 0.04 (b); both cases refer to a single interaction point and to $\mathrm{LHC}$ injection at $450 \mathrm{GeV} / c$. artificially held constant and equal to the rms beam size $\sigma_{b}$, whereas for the more realistic narrowly spiked nonGaussian electron distribution, the size of the beam core increases. The core growth arises due to a different mechanism; i.e., near the tail of the bunch, the tune shift on the beam axis can be so large that the motion becomes linearly unstable, which leads to a blow up of the transverse beam core in the corresponding $z$ regions. Since beam particles perform synchrotron motion, they cross the unstable region twice per turn, which amounts to a "periodic crossing of instability." Linear instability can occur if the electron cloud is not uniformly distributed but concentrated in specific locations around the accelerator, e.g., inside a certain type of lattice element. If the cloud distribution and its electrostatic potential around the ring and along the bunch are known, the stability of the beam core can be determined analytically by computing the trace of the 1-turn transport matrix, $M(z)$, including the linear electron-cloud force around the ring.

Concentrating all electrons of the ring in a single electron-beam interaction point (either as a computational simplification or because the electrons are, in fact, confined to a single short section of the ring) excites all possible resonances and leads to maximum linear instability. For such a case and considering a high average electron density of $\rho_{e}=10^{14} \mathrm{~m}^{-3}$, Fig. 4 shows phase-space trajectories at different positions $z$ and the corresponding frequency spectra along the bunch, obtained from a simulation without synchrotron motion. Here, at each $z$ position, a single particle was launched close to the origin in order to probe the stability of the $z$-dependent closed orbit. The linear instability leads to the emergence of a hyperbolic fixed point near the longitudinal bunch center. For larger values of $z$ (in the bunch tail) the motion appears to be chaotic, as is strongly suggested by the wide frequency spread in the corresponding spectrum. The $z$ position where the transition from linearly stable to unstable motion occurs depends on the electron distribution around the ring, the linear tune shift induced by the electron cloud, and the unperturbed betatron tune.

As simulations with the analytical field model give results similar to the full-blown PIC code (Fig. 3), we adopt the former for studying a more accurate model of the SPS. Namely, instead of considering a few artificial electron-beam interaction points per turn, we include interaction points in all cells of the optical lattice, separated by a distance much smaller than the betatron wavelength. Resonances are still excited since we assume, as in reality, that the electron cloud is formed mainly in the dipole magnets [6], which are distributed nonuniformly around the ring. Specifically, we tracked 1000 proton macroparticles through the full SPS optics with 4152 elements per turn, including 744 beam-electron interaction points, one at each SPS dipole, for standard SPS beam parameters (Table III of [11]) and a maximum incoherent tune shift of $\Delta Q \approx 0.13$, due to the pinched electrons. A large chromaticity of $\left|Q_{y}^{\prime} / Q_{y}\right| \approx 1$ was included, as it is introduced 

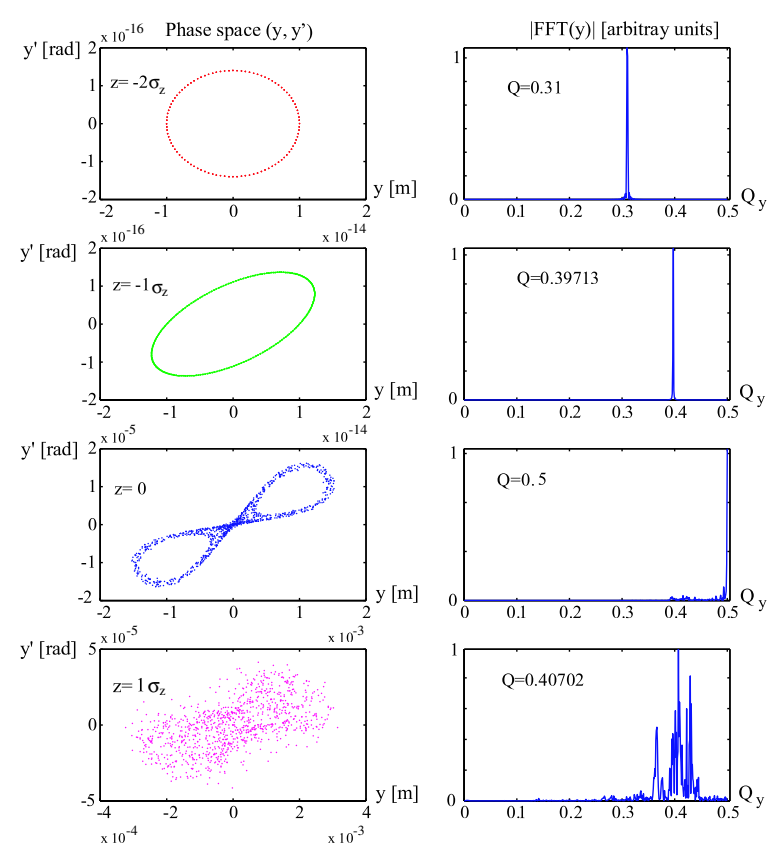

FIG. 4 (color online). Vertical phase space and frequency spectrum of a particle launched at an initial transverse amplitude of $10^{-14} \mathrm{~m}$, close to the origin, for different $z$ positions, illustrating the transition from an elliptical to a hyperbolic fixed point. Note the variation in scale for different values of $z$. The initial average electron density around the ring is $\rho_{e} \approx$ $10^{14} \mathrm{~m}^{-3}$, and the electron-beam interaction is concentrated at a single location of the ring. The simulation was performed for the LHC at injection with a beam momentum of $450 \mathrm{GeV} / c$.

in the real SPS for preventing coherent electron-cloud instabilities. The nonzero chromaticity leads to resonance crossing by an increased number of bunch particles, as in the case of space charge [16]. The beam pipe radius was taken to be $2.5 \mathrm{~cm}$ (constant around the ring), corresponding to a realistic minimum aperture of $6 \sigma_{b}$. The emittance growth simulated with this model varies between $10 \%$ and $40 \%$ over $5 \times 10^{5}$ turns, or about $10 \mathrm{~s}$, depending on plane
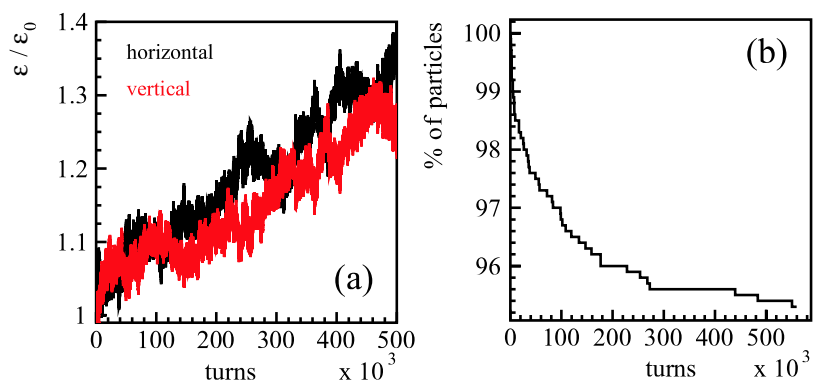

FIG. 5 (color online). Simulated emittance growth (a) and losses (b) as a function of turn number with electron-beam interactions at each of the 744 SPS dipoles, for $26 \mathrm{GeV} / c, Q_{x}=$ 26.18, $Q_{y}=26.15,\left|Q_{x, y}^{\prime}\right| \approx 26$ (and using 1000 proton macroparticles). and working point. Over the same time, the simulations show up to $5 \%$ beam loss. Figure 5 presents a typical result.

The simulated emittance growth and losses are larger for tunes at which SPS experiments indeed exhibited shorter beam lifetimes. Simulations for other tunes, e.g., $Q_{x} \approx$ 26.38 , indicate that also the electron-induced linear instability may occur in the SPS.

We have discussed two mechanisms likely responsible for incoherent emittance growth in proton or positron storage rings, which may explain observations at several operating accelerators, and which could set much tighter tolerances on acceptable electron densities for future ones than previously assumed.

The authors thank G. Arduini, H. Fukuma, K. Ohmi, K. Oide, F. Ruggiero, G. Rumolo, D. Schulte, and E. Shaposhnikova for fruitful discussions.

[1] (a) Proceedings of ECLOUD'02: Mini-Workshop on Electron-Cloud Simulations for Proton and Positron Beams, Geneva, Switzerland, 2002, edited by G. Rumolo and F. Zimmermann (CERN Report No. CERN-2002-001, 2002); (b) Proceedings of ECLOUD'04: Advanced ICFA Beam Dynamics Workshop on Electron-Cloud Effects, Napa, California, 2004, edited by M. Furman, S. Henderson, and F. Zimmermann (CERN Report No. CERN-2005-001, 2005).

[2] K. Ohmi, Phys. Rev. Lett. 75, 1526 (1995).

[3] K. Ohmi and F. Zimmermann, Phys. Rev. Lett. 85, 3821 (2000).

[4] K. Ohmi, F. Zimmermann, and E. Perevedentsev, Phys. Rev. E 65, 016502 (2001).

[5] E. Shaposhnikova (private communication).

[6] J. M. Jiménez et al., in Ref. [1(a)].

[7] H. Fukuma, in Ref. [1(b)].

[8] J. Wei et al., in Proceedings of the 2005 Particle Accelerator Conference (PAC), Knoxville, Tennessee, 2005, edited by C. Horak (IEEE, Piscataway, NJ, 2005), p. 4087.

[9] X. Zhang (private communication).

[10] G. Rumolo et al., Phys. Rev. ST Accel. Beams 5, 121002 (2002).

[11] E. Benedetto et al., Phys. Rev. ST Accel. Beams 8, 124402 (2005).

[12] M. A. Furman and A. A. Zholents, in Proceedings of the 1999 Particle Accelerator Conference (PAC), New York, 1999, edited by A. Luccio and W. Mackay (IEEE, Piscataway, NJ, 1999), p. 1794.

[13] A. W. Chao and M. Month, Nucl. Instrum. Methods 121, 129 (1974).

[14] B. V. Chirikov et al., Physica (Amsterdam) 14D, 289 (1985).

[15] G. Franchetti et al., in Proceedings of HHH-2004, Geneva, Switzerland, 2004 (CERN Report No. CERN-2005-005, 2005).

[16] G. Franchetti and I. Hofmann, Nucl. Instrum. Methods Phys. Res., Sect. A 561, 195 (2006). 